\title{
A 34-Year-Old Woman with Brainstem Cavernous Malformation: The Anterior Transcallosal Transchoroidal Approach and Literature Review
}

\author{
Sayied Abdol Mohieb Hosainey ${ }^{1}$ Torstein R. Meling ${ }^{1}$ \\ ${ }^{1}$ Department of Neurosurgery, Oslo University Hospital, \\ Rikshospitalet, Oslo, Norway \\ Address for correspondence Torstein R. Meling, MD, PhD, \\ Department of Neurosurgery, Oslo University Hospital, Rikshospitalet, \\ $\mathrm{N}-0027$ Oslo, Norway (e-mail: torsteinrmeling@mailcity.com; \\ J Neurol Surg Rep 2014;75:e236-e240. \\ torstein.meling@rikshospitalet.no).
}

\author{
Abstract \\ Keywords \\ - cavernous \\ malformation \\ - brainstem cavernoma \\ - surgical approach \\ - transcallosal \\ transchoroidal \\ approach \\ - surgical \\ complications \\ - brainstem \\ hemorrhage
}

Mesencephalic cavernous malformations (MeCMs) account for 4 to $35 \%$ of the cavernous malformations of the central nervous system and are generally rare. Surgical resection of brainstem cavernomas are high-risk procedures and can be challenging to the neurosurgeon. Several approaches have been described, but the approach must allow for a straight line of sight in which the surgeon, the pial incision, and the MeCM are all collinear. This alignment provides the best view of the lesion while minimizing the need for brainstem retraction. The pial incision should be chosen to minimize the distance to the lesion while avoiding critical nuclei and tracts. In this case report, we present a 34-year-old woman with a MeCM resected by an anterior transcallosal transchoroidal approach with minimal damage to surrounding brain tissue. Although rarely used, it should be considered a valuable alternative to ventrally located brainstem cavernomas.

\section{Introduction}

Cavernous malformations (CMs) of the central nervous system have an estimated prevalence of $<1 \%$ in the general population. ${ }^{1,2}$ Brainstem cavernomas account for 4 to $35 \%$ of intracranial cavernomas in contemporary series. ${ }^{2-5}$ These lesions may give rise to severe and complex neurologic deficits if they hemorrhage. However, surgery within this region is also not without inherent risks. Irreversible deficits due to damage of sensitive brainstem structures are important factors to be taken into careful consideration. If surgery is proposed, it requires the utmost planning. Depending on location, different surgical approaches to brainstem CMs have been performed and reported in contemporary studies. We report a case of a young healthy woman presenting with a brainstem CM that was resected by an anterior transcallosal transchoroidal approach (ATTA), an approach rarely used but that promises a valuable alternative to ventral mesencephalic CMs (MeCMs).

\section{Case Presentation}

\section{History}

A 34-year-old woman, with no prior history of comorbidities, contacted her general practitioner because of double vision. She was referred to an ophthalmologist and prescribed optical lenses. Due to persistent double vision and inability to focus, she was referred to a cerebral magnetic resonance imaging (MRI) scan a year later. The MRI showed a mesencephalic CM posterior to the interpeduncular cistern. No measures were initially taken due to its small size and highly eloquent location. Regular controls at the outpatient clinic showed no progression of symptoms or increased tumor size until mid-summer 2012. She then developed dizziness, wors-
License terms Stuttgart · New York

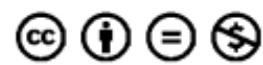

received

April 26, 2014

accepted

June 15, 2014

published online

August 21, 2014
DOI http://dx.doi.org/

10.1055/s-0034-1387192. ISSN 2193-6358. 

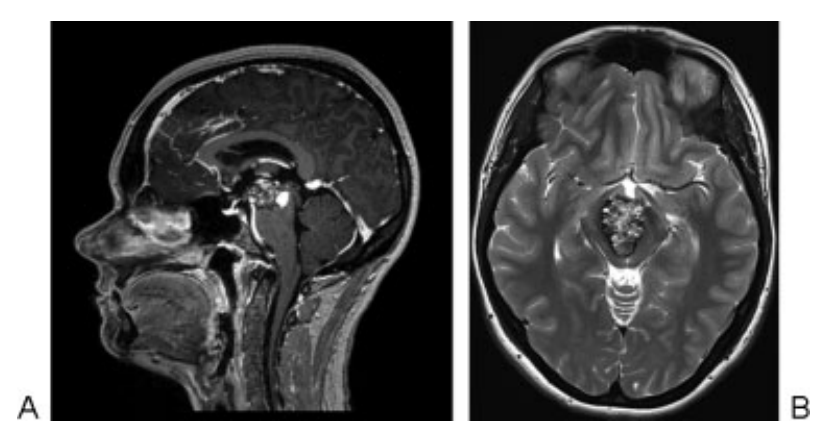

Fig. 1 Magnetic resonance imaging (MRI) of the brainstem cavernoma located in the mesencephalon posterior to the interpeduncular cistern and extending into the third ventricle. (A) Sagittal view of T1-weighted MRI with contrast media. (B) Axial T2-weighted MRI without contrast media.

ening of her double vision, and imbalance. A new MRI showed progression of the MeCM ( $\mathbf{- F i g . ~ 1 A , ~ B ) , ~ a n d ~ s h e ~ w a s ~ r e f e r r e d ~}$ to our neurosurgical department. The neurologic examination showed diplopia, internuclear ophthalmoplegia with obvious affection of the occulomotor nerve, semidilated pupil of the right eye with slow pupillary light reflex, and impaired accommodation. There were no signs of ataxia or other gait disturbances. However, she continued to deteriorate, with worsening of the symptoms just mentioned, and developed a generalized headache $\sim 1$ month later. She was therefore offered surgery (T. R. M.).

\section{Surgical Technique}

Because the MeCM reached the surface of the third ventricle and the pyramidal tracts were pushed quite symmetrically laterally (-Fig. 2), we opted for an approach reaching the lesion from above. Using the ATTA, the patient was put in a park bench position left side up and with the sagittal suture

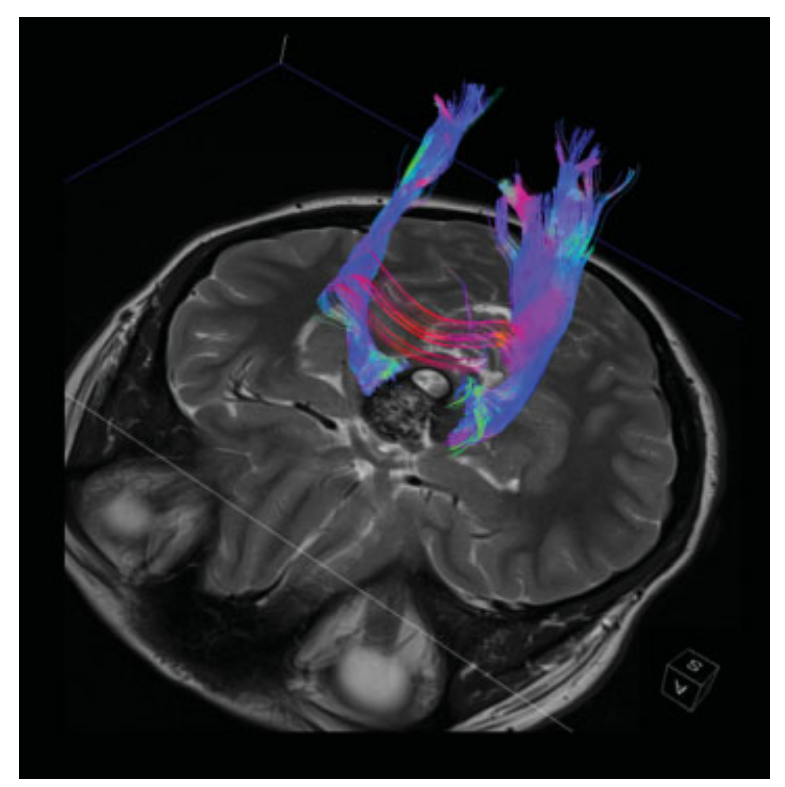

Fig. 2 Diffusion tensor imaging with tractography showing the white pyramidal tracts surrounding the mesencephalic cavernous malformation. aligned with the horizontal plane. This allows gravity to pull on the lower hemisphere and open up the interhemispheric fissure, obviating the need for retractors. Because the MeCM surfaced in the posterior third ventricle, an anterior-toposterior angle of approach was planned. Neuronavigation and neurophysiologic intraoperative monitoring of motorevoked potentials and somatosensory-evoked potentials were used. A modified bicoronal skin incision was used, running in between the temporal lines bilaterally. The craniotomy was placed using neuronavigation to avoid sacrificing any bridging veins. A $5 \times 7$-cm craniotomy eccentric to the midline was placed, exposing the right frontal superior gyrus and the sagittal sinus. The dura was opened in between two bridging veins and pulled upward to retract the superior sagittal sinus. Dissection through the interhemispheric fissure allowed identification of the callosomarginal and pericallosal arteries. An anteriorly located callosotomy of $15 \mathrm{~mm}$ enabled further entry into the anterior horn of the right lateral ventricle with subsequent visualization of the foramen of Monro. Once inside the lateral ventricle, the choroid plexus was dissected from the fornix and retracted laterally. In this way, the fornix was spared. To improve access into the third ventricle, the right septal vein was coagulated and cut. The thalamostriate vein, along with the other veins supplying the lateral ventricle including the choroid plexus, were preserved. Further dissection of the choroidal fissure to gain access to the velum interpositum allowed identification of the internal cerebral veins. These were dissected free from surrounding tissue within the velum interpositum back toward the habenula and corpus pineale and subsequently retracted laterally. Through the velum interpositum, the third ventricle was entered and the $\mathrm{MeCM}$ could be easily visualized. Laterally, on the left and right side of the cavernoma, deep arteries were dissected free from the tumor under microscopic guidance. A deep Rosenthal vein perforating straight through the middle of the cavernoma was also dissected free and spared. The cavernoma was circumferentially dissected from the surrounding brainstem and removed in a piecemeal fashion. Lastly, in the depth of the cavity, three perforating arteries were dissected free, and complete extirpation of the cavernoma was achieved without damage to surrounding brain tissue.

\section{Postoperative Outcome}

Immediate postoperative status showed no new-onset neurologic deficits, and complete resection of the mesencephalic cavernoma was achieved ( - Fig. 3A, B). The preoperative internuclear ophthalmoplegia, pupillary asymmetry with slow pupillary light reflex, and diplopia were still present after surgery.

\section{Literature Review}

Neurosurgical approaches to brainstem CMs can be divided into three groups based on angle of surgical approach: the anterior/anterolateral approaches, the superior approaches and the posterior/posterolateral approaches. Within each of these groups are variations of the approaches. 

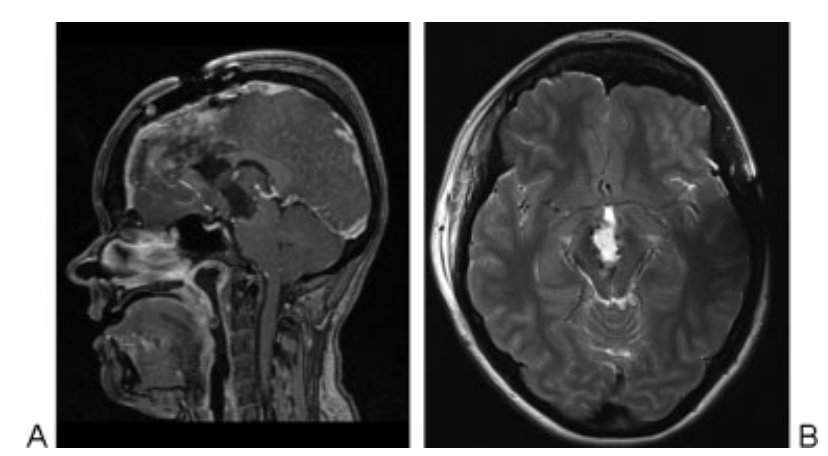

Fig. 3 Postoperative magnetic resonance imaging (MRI) showing total resection of the mesencephalic cavernous malformation. (A) Sagittal view of T1-weighted MRI with contrast media. (B) Axial T2-weighted MRI without contrast media.

The anterior/anterolateral approaches, for example, the frontotemporal orbitozygomatic (FTOZ) approach, the subtemporal, and pterional approaches are generally used for MeCMs in the interpeduncular cistern or anterolaterally located lesions. Depending on the location of the lesion, these approaches have various advantages. For instance, the FTOZ with a transsylvian approach provides the surgeon with a flatter view of the midbrain than a pterional approach. ${ }^{6}$ In a study by Abla et al including 260 patients with brainstem CMs, 18 patients had an FTOZ approach, 6 patients had a subtemporal approach with or without anterior petrosectomy, and one underwent a pterional approach. ${ }^{7}$ Other approaches such as the transotic, transpetrosal, transcochlear, and translabyrinthine approaches are rarely used due to their inherent morbidity and complexity, but they provide a wider and more lateral exposure for the lower midbrain, pons, and upper medulla with lesions involving ventrolateral medulla extending caudally. ${ }^{6,8-10}$ Nevertheless, it is important to bear in mind complications such as cerebrospinal fluid (CSF) leakage, auditory damage, and cranial nerve deficits. Finally, the transoral-transclival approach can be used to gain full access to the anterolateral medullary lesions and to the cisternal space around it, but it has a high associated morbidity with risks of postoperative CSF fistulas and infections. ${ }^{11}$

The superior approaches can be subdivided into anterior and posterior (occipital) interhemispheric approaches. The contralateral transcallosal approach, in which the patient is positioned with the head horizontally to allow for gravity to pull the dependent hemisphere toward the floor to obviate the use of retractors, is a variant. ${ }^{12}$ For access to the brainstem, the superior interhemispheric approaches are followed by a transcallosal-transchoroidal step to enter the third ventricle. ${ }^{13-15}$ In published series, the superior approaches are rarely used but are quite suitable for lesions in the upper midbrain region with or without extension to the region of the third ventricle and/or to surrounding medial structures in the basal ganglia. In a study of surgical management of brainstem cavernomas in 36 consecutive patients by Ohue et al, only 4 patients had an occipital transtentorial approach for lesions in the mesencephalon. ${ }^{5}$ In their study of 79 patients with eloquently based CMs, Chang et al had only two patients who underwent a transcallosal-transchoroidal approach, which provided excellent exposure of the medial thalamus through the third ventricle. ${ }^{16,17}$ Ferroli et al, in their study of 52 patients with brainstem CMs, only one patient with a mesencephalothalamic lesion underwent a transcallosal surgical approach. ${ }^{18}$ However, limitations such as the columns of the fornix limit the access to the anterior third ventricle, and the approach may also put critical veins at risk. $^{19}$

The posterior approaches are generally the most commonly used approaches for brainstem CMs because of the higher incidences of brainstem cavernomas located in the pontine and medullary regions.,20,21 The suboccipital approach, supracerebellar infratentorial approach (median, paramedian, far lateral. and extreme lateral), and the retrosigmoid approach all belong to this group. In the previously mentioned study by Abla et al with 260 patients with brainstem CMs, 74 patients underwent a suboccipital approach. ${ }^{7}$ Of the 52 patients in the study by Ferroli et al, 31 patients underwent a suboccipital approach. ${ }^{18}$ These approaches allow for an adequate view of the posterior and posterolateral surface of the midbrain and quadrigeminal plate, as well as the posterolateral surface of the upper pons and ventrolateral medullary region. ${ }^{6,22}$ Other rare approaches have also been reported that include the telovelar (transcerebellar-medullary) approach, the transvermian approach, the far lateral transcondylar approach, and the tonsillouveal transaqueductal approach that sometimes involve removal of the cervical vertebrae to access the pontomedullary region. ${ }^{22-25}$ The disadvantages are risks of damage to the lower cranial nerves exiting from the pontomedullary junctions with subsequent neurologic deficits. ${ }^{1,7,26}$

\section{Discussion}

Brainstem CMs often represent a considerable microsurgical challenge to the neurosurgeon, mainly because of location, but also due to the high risk of complications such as neurologic deficits and residual tumor. Estimates of annual hemorrhage rate from a brainstem cavernoma with no history of prior hemorrhage ranges from 0.5 to $6 \%$ per patient year, 2,7,20,27 and the morbidity related to a bleeding is often severe. The indications for surgery on brainstem CMs are severe or repeat minor bleedings and/or deteriorating neurologic function., ${ }^{5,28}$ Furthermore, to prevent patients' functional decline owing to recurrent events and to reduce the surgical morbidity, early surgery is often recommended. ${ }^{28,29}$ Although our patient did not present with acute symptoms, surgery was indicated due to progressive worsening.

The surgical approach to MeCMs are mainly chosen based on ease of direct access to the lesion, with no or minimal damage to brain tissue ( - Table 1 ). The surgeon must not only consider the shortest path from the surface, but also the safest path. This makes a detailed knowledge of the brainstem neuroanatomic pathways of critical importance. ${ }^{1,6,27}$ In the preoperative planning stage, MRI with diffusion tensor 
Table 1 Location versus surgical approaches for brainstem cavernomas

\begin{tabular}{|l|l|l|}
\hline & Location & Approach \\
\hline Mesencephalon & Anterior & $\begin{array}{l}\text { FTOZ } \\
\text { Pterional } \\
\text { Subtemporal } \\
\text { Interhemispheric }\end{array}$ \\
\hline & Posterior & $\begin{array}{l}\text { Supracerebellar infratentorial (median, paramedian, } \\
\text { and extreme lateral) } \\
\text { Occipital transtentorial/interhemispheric }\end{array}$ \\
\hline Pons & Anterior & $\begin{array}{l}\text { Retrosigmoid } \\
\text { Subtemporal transtentorial } \\
\text { Presigmoid } \\
\text { Transpetrosal }\end{array}$ \\
& & $\begin{array}{l}\text { Transotic } \\
\text { Transcochlear } \\
\text { Translabyrinthine }\end{array}$ \\
& & Suboccipital \\
& & $\begin{array}{l}\text { Telovelar } \\
\text { Transvermian }\end{array}$ \\
\hline Medulla oblongata & Posterior & $\begin{array}{l}\text { Retrosigmoid } \\
\text { Suboccipital }\end{array}$ \\
& & Far lateral \\
& & Transoral \\
& & Tonsillouveal transaqueductal \\
\hline
\end{tabular}

Abbreviation: FTOZ, frontotemporal orbitozygomatic.

imaging and white matter tractography was used because it has shown to be beneficial for superior quantification and visualization of brainstem lesions ${ }^{30}$ (-Fig. 2).

Generally, most dorsally located MeCMs can be accessed via a supracerebellar infratentorial approach (midline, paramedian, far lateral, or an extreme lateral approach, depending on the exact location of the cavernoma); anteriorly located brainstem CMs are usually accessed via an orbitozygomatic (OZ), subtemporal, or pterional transsylvian approach. ${ }^{1,3,6,22}$

The MeCM in our patient was ventrally located and just posterior to the interpeduncular cistern (-Fig. 1A, B). Consequently, an FTOZ, OZ, or pterional transsylvian approach could have been used. ${ }^{6}$ However, the ATTA was used to avoid damage to surrounding brain tissue with respect to the pyramidal tracts surrounding the cavernoma. A subtemporal pterional transsylvian or $\mathrm{OZ}$ approach seemed inappropriate because the risk of damaging the tracts laterally located to the tumor was probable. Although a $15-\mathrm{mm}$ callosotomy was done, an approach from above the tumor seemed better because the superior surface of the cavernoma extending into the third ventricle was devoid of brain tissue and clearly visible. Partial sectioning of the corpus callosum does not cause significant neurologic deficits. ${ }^{31}$ Bertalanffy has suggested an anterior interhemispheric approach from the forehead, where the anterior communicating artery (ACOM) is cut to access the mesencephalic cavernoma (personal communication). Although the distance will be shorter with this approach, cutting the ACOM artery may disturb or abolish the blood supply to the structures nearby such as the optic chiasma, lamina terminalis, hypothalamus, anterior columns of the fornix, and paraolfactory areas. Additionally, with this approach through the lamina terminalis to access the third ventricle, nearby sensitive structures such as the optic chiasma are at risk of being damaged.

According to the current literature, the ATTA is rarely used. However, it can be applied to CMs that extend into the third ventricle. Apart from the microsurgical technicalities, we believe that five steps of the procedure are important to consider when using the ATTA. (1) Proper positioning of the patient to allow adequate brain relaxation and easy access to the third ventricle because this is critically important to minimize retraction-induced injury and should occur before the interhemispheric dissection. ${ }^{17}$ (2) Placement of the craniotomy with respect to the angle of approach. (3) Verification of which lateral ventricle that has been entered is done by identifying the thalamostriate vein and foramen of Monro; if the vein is to the right of the foramen of Monro, the right lateral ventricle has been entered. ${ }^{19}(4)$ The dissection of the choroidal fissure to release the choroid plexus from the fornix must be performed extremely carefully. (5) Lastly, the velum interpositum must be opened widely, from anterior all the way to the habenula.

\section{Conclusion}

Brainstem CMs continue to present a considerable microsurgical challenge. Most ventral MeCMs can be approached via a transsylvian or a FTOZ approach. Although rarely used, the ATTA can serve as a valuable alternative approach to ventral MeCMs. 


\section{Financial Disclosure}

The authors have nothing to disclose.

\section{References}

1 Bertalanffy H, Benes L, Miyazawa T, Alberti O, Siegel AM, Sure U. Cerebral cavernomas in the adult. Review of the literature and analysis of 72 surgically treated patients. Neurosurg Rev 2002; 25(1-2):1-53; discussion 54-55

2 Dukatz T, Sarnthein J, Sitter H, et al. Quality of life after brainstem cavernoma surgery in 71 patients. Neurosurgery 2011;69(3): 689-695

3 Gross BA, Batjer HH, Awad IA, Bendok BR. Brainstem cavernous malformations. Neurosurgery 2009;64(5):E805-E818; discussion E818

4 Gross BA, Batjer HH, Awad IA, Bendok BR, Du R. Brainstem cavernous malformations: 1390 surgical cases from the literature. World Neurosurg 2013;80(1-2):89-93

5 Ohue S, Fukushima T, Kumon Y, Ohnishi T, Friedman AH. Surgical management of brainstem cavernomas: selection of approaches and microsurgical techniques. Neurosurg Rev 2010;33(3): 315-322; discussion 323-324

6 Giliberto G, Lanzino DJ, Diehn FE, Factor D, Flemming KD, Lanzino G. Brainstem cavernous malformations: anatomical, clinical, and surgical considerations. Neurosurg Focus 2010;29(3):E9

7 Abla AA, Lekovic GP, Turner JD, de Oliveira JG, Porter R, Spetzler RF. Advances in the treatment and outcome of brainstem cavernous malformation surgery: a single-center case series of 300 surgically treated patients. Neurosurgery 2011;68(2):403-414; discussion 414-415

8 Horgan MA, Delashaw JB, Schwartz MS, Kellogg JX, Spektor S, McMenomey SO. Transcrusal approach to the petroclival region with hearing preservation. Technical note and illustrative cases. J Neurosurg 2001;94(4):660-666

9 Kawase T, Shiobara R, Toya S. Anterior transpetrosal-transtentorial approach for sphenopetroclival meningiomas: surgical method and results in 10 patients. Neurosurgery 1991;28(6):869-875,; discussion 875-876

10 Miller CG, van Loveren HR, Keller JT, Pensak M, el-Kalliny M, Tew JM Jr. Transpetrosal approach: surgical anatomy and technique. Neurosurgery 1993;33(3):461-469; discussion 469

11 Reisch R, Bettag M, Perneczky A. Transoral transclival removal of anteriorly placed cavernous malformations of the brainstem. Surg Neurol 2001;56(2):106-115; discussion 115-116

12 Spetzler RF, Sanai N. The quiet revolution: retractorless surgery for complex vascular and skull base lesions. J Neurosurg 2012;116(2): 291-300

13 Apuzzo ML, Chikovani OK, Gott PS, et al. Transcallosal, interfornicial approaches for lesions affecting the third ventricle: surgical considerations and consequences. Neurosurgery 1982;10(5):547-554

14 Tomasello F, Cardali S, Angileri FF, Conti A. Transcallosal approach to third ventricle tumors: how I do it. Acta Neurochir (Wien) 2013; 155(6):1031-1034
15 Wen HT, Rhoton AL Jr, de Oliveira E. Transchoroidal approach to the third ventricle: an anatomic study of the choroidal fissure and its clinical application. Neurosurgery 1998;42(6):1205-1217; discussion 1217-1219

16 Chang EF, Gabriel RA, Potts MB, Berger MS, Lawton MT. Supratentorial cavernous malformations in eloquent and deep locations: surgical approaches and outcomes. Clinical article. J Neurosurg 2011;114(3):814-827

17 Kasowski HJ, Nahed BV, Piepmeier JM. Transcallosal transchoroidal approach to tumors of the third ventricle. Neurosurgery 2005; 57 (4, Suppl):361-366; discussion 361-366

18 Ferroli P, Sinisi M, Franzini A, Giombini S, Solero CL, Broggi G. Brainstem cavernomas: long-term results of microsurgical resection in 52 patients. Neurosurgery 2005;56(6):1203-1212; discussion $1212-1214$

19 Ulm AJ, Russo A, Albanese E, et al. Limitations of the transcallosal transchoroidal approach to the third ventricle. J Neurosurg 2009; 111(3):600-609

20 Porter RW, Detwiler PW, Spetzler RF, et al. Cavernous malformations of the brainstem: experience with 100 patients. J Neurosurg 1999;90(1):50-58

21 Steinberg GK, Chang SD, Gewirtz RJ, Lopez JR. Microsurgical resection of brainstem, thalamic, and basal ganglia angiographically occult vascular malformations. Neurosurgery 2000;46(2): 260-270; discussion 270-271

22 de Oliveira JG, Lekovic GP, Safavi-Abbasi S, et al. Supracerebellar infratentorial approach to cavernous malformations of the brainstem: surgical variants and clinical experience with 45 patients. Neurosurgery 2010;66(2):389-399

23 Dammers R, Delwel EJ, Krisht AF. Cavernous hemangioma of the mesencephalon: tonsillouveal transaqueductal approach. Neurosurgery 2009;64(5, Suppl 2):296-299; discussion 299-300

24 Garber ST, Couldwell WT. Resection of an inferior pontine cavernous malformation using a far-lateral transcondylar approach. Acta Neurochir (Wien) 2011;153(12):2461-2464

25 Mussi AC, Rhoton AL Jr. Telovelar approach to the fourth ventricle: microsurgical anatomy. J Neurosurg 2000;92(5):812-823

26 Chen L, Zhao Y, Zhou L, Zhu W, Pan Z, Mao Y. Surgical strategies in treating brainstem cavernous malformations. Neurosurgery 2011; 68(3):609-620; discussion 620-621

27 Garrett M, Spetzler RF. Surgical treatment of brainstem cavernous malformations. Surg Neurol 2009;72(Suppl 2):S3-S9; discussion S9-S10

28 Wang CC, Liu A, Zhang JT, Sun B, Zhao YL. Surgical management of brain-stem cavernous malformations: report of 137 cases. Surg Neurol 2003;59(6):444-454; discussion 454

29 Hauck EF, Barnett SL, White JA, Samson D. Symptomatic brainstem cavernomas. Neurosurgery 2009;64(1):61-70; discussion 70-71

30 Chen X, Weigel D, Ganslandt O, Buchfelder M, Nimsky C. Diffusion tensor imaging and white matter tractography in patients with brainstem lesions. Acta Neurochir (Wien) 2007;149(11): 1117-1131; discussion 1131

31 Kasowski H, Piepmeier JM. Transcallosal approach for tumors of the lateral and third ventricles. Neurosurg Focus 2001;10(6):E3 